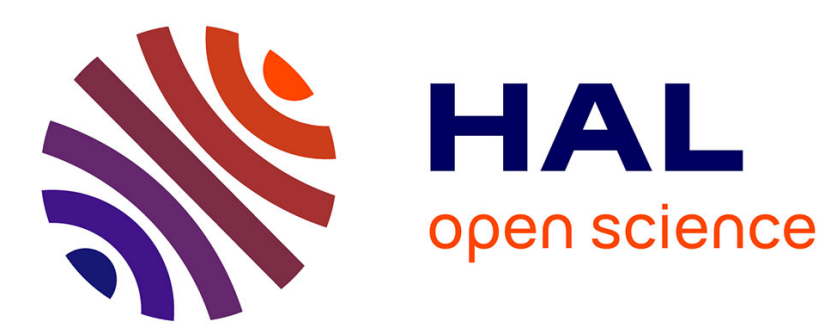

\title{
Convergence conditions for Persidskii systems
}

Wenjie Mei, Denis Efimov, Rosane Ushirobira, Alexander Aleksandrov

\section{To cite this version:}

Wenjie Mei, Denis Efimov, Rosane Ushirobira, Alexander Aleksandrov. Convergence conditions for Persidskii systems. ECC 2021 - 19th European Control Conference, Jun 2021, Rotterdam / Virtual, Netherlands. hal-03255877

\section{HAL Id: hal-03255877 \\ https://hal.inria.fr/hal-03255877}

Submitted on 9 Jun 2021

HAL is a multi-disciplinary open access archive for the deposit and dissemination of scientific research documents, whether they are published or not. The documents may come from teaching and research institutions in France or abroad, or from public or private research centers.
L'archive ouverte pluridisciplinaire HAL, est destinée au dépôt et à la diffusion de documents scientifiques de niveau recherche, publiés ou non, émanant des établissements d'enseignement et de recherche français ou étrangers, des laboratoires publics ou privés. 


\title{
Convergence conditions for Persidskii systems
}

\author{
Wenjie $\mathrm{Mei}^{1}$, Denis Efimov ${ }^{1,2}$, Rosane Ushirobira ${ }^{1}$, Alexander Aleksandrov ${ }^{3}$
}

\begin{abstract}
A class of generalized Persidskii systems is considered in this work. The conditions of convergence for Persidskii systems are introduced, which can be checked through linear matrix inequalities. Also, the case of almost periodic convergence of this class of dynamics with almost periodic input is studied. The proposed results are applied to a Lotka-Volterra model.
\end{abstract}

\section{INTRODUCTION}

If a dynamical system is convergent, i.e., it has a unique and bounded asymptotically stable solution [1], [2], then computation of only one trajectory is sufficient to determine the asymptotic behavior of all others. This property is similar to incremental stability [3], [4] (skipping the requirement on boundedness), and the contraction theory is frequently used for its analysis [5]. The convergence property has received increased interest due to its usefulness in system analysis and design, e.g., it excludes the case of several co-existing solutions for nonlinear output regulation [6], synchronization [7], [8], [9], steady-state analysis of nonlinear systems [10] and many other control problems.

It is evident that not all nonlinear systems are convergent: the trajectories may be convergent for some (e.g., zero) inputs and not for others, and an example of such a behavior is demonstrated by Duffing's model [11], in which multiple limit cycles phenomena and sub-harmonic oscillations may occur. Thus, for nonlinear systems, it is required to consider additional conditions for investigating convergence property.

We may additionally ask for conditions of preservation by the attracting solution of the (almost) periodicity of the input [12], [13]. Note that in nonlinear systems, the frequency of entrainment is not necessarily kept by the output [14], and a typical example is again Duffing's model, which can generate chaotic trajectories under harmonic excitation. The relations between the existence of almost periodic solutions and properties of the right-hand sides of dynamical systems have been studied for centuries (see, e.g., Markov's theorem [15]).

*This work was partially supported by the China Scholarship Council (CSC) Scholarship (CSC No. 201908050104), by the Ministry of Science and Higher Education of Russian Federation, passport of goszadanie no. 2019-0898, and by the Russian Foundation for Basic Research (grant no. 19-01-00146-a).

${ }^{1}$ Wenjie Mei, Denis Efimov, and Rosane Ushirobira are with Inria, Univ. Lille, CNRS, UMR 9189 - CRIStAL, F59000 Lille, France \{Wenjie.Mei, Denis.Efimov, Rosane.Ushirobira\} ainria.fr

${ }^{2}$ Denis Efimov is with ITMO University, 49 av. Kronverkskiy, 197101 Saint Petersburg, Russia

${ }^{3}$ Alexander Aleksandrov is with Saint Petersburg State University, Saint Petersburg, 199034, Russia a.u.aleksandrovespbu.ru
The property of convergence also has a rich history of investigation. For example, Pliss [16] investigated the convergence of the system $\dot{x}=f(t, x)$ with a periodic righthand side. Later, Demidovich [17] extended the special case analyzed by Pliss to a more general $f$. In the same vein, Zubov [18], [19] established a periodic/almost periodic convergence criterion for nonlinear systems. There are also further explorations of convergence properties for nonlinear systems, e.g., convergence-like properties by Lyapunov's second method [20], sufficient conditions of convergence of the system with a scalar nonlinearity satisfying an incremental sector condition [21], the convergence of perturbed nonlinear systems [22], [3], and convergence conditions of a class of nonlinear systems [23].

In this work, we study the convergence property and the existence of almost periodic trajectories for a class of Persidskii systems, which was introduced in [24], [25] as $\dot{x}=A_{0} x+A_{1} f(x)$. Recently, its generalized version was investigated in [26], [27] by taking into account multiple nonlinearities: $\dot{x}=A_{0} x+\sum_{i=1}^{M} A_{i} f_{i}(x)$ (more detailed definition is given below). The advantage of this class of models is that despite its significant nonlinearity (with $M \geq 1$ ), a Lyapunov function is suggested in the literature such that the analysis of its properties can be performed by solving linear matrix inequalities, which is a constructive approach.

The organization of this paper is as follows. In Section II, the preliminaries and the definitions of utilized stability properties are presented. The class of considered systems is defined in Section III. The conditions for convergence and almost periodic convergence of generalized Persidskii systems are given in Section IV. The model of Lotka-Volterra is considered an application for examining the efficiency of the proposed results in Section V.

\section{NOTATION}

- $\mathbb{N}, \mathbb{Z}$ and $\mathbb{R}$ represent the sets of natural numbers, integers and real numbers, respectively, $\mathbb{R}_{+}=$ $\{s \in \mathbb{R}: s \geq 0\} ; \mathbb{R}^{n}\left(\mathbb{R}_{+}^{n}\right)$ and $\mathbb{R}^{m \times n}$ denote the vector spaces of $n$-tuples of real numbers (nonnegative real numbers) and $m \times n$ real matrices, respectively. The symbol $\|\cdot\|$ refers to the Euclidean norm on $\mathbb{R}^{n}$ (and the induced matrix norm $\|A\|$ for a matrix $A \in \mathbb{R}^{m \times n}$ ).

- For $p, n \in \mathbb{N}$ with $p \leq n$, the notation $\overline{p, n}$ is used to represent the set of nonnegative integers $\{p, \ldots, n\}$.

- The $n \times n$ diagonal matrix with $v \in \mathbb{R}^{n}$ on the main diagonal is denoted by $\operatorname{diag}(v)$. Let $v>0(v<0)$ indicate $v_{i}>0\left(v_{i}<0\right)$ for $i \in \overline{1, n}$.

- The transpose of a matrix $A \in \mathbb{R}^{n \times n}$ is denoted by $A^{\top}$. Let $\lambda_{\min }(A)\left(\lambda_{\max }(A)\right)$ refer to the minimal (maximal) 
eigenvalue of a symmetric matrix $A$, and $I_{n}$ correspond to the $n \times n$ identity matrix. Denote the vector of dimension $n$ with all elements equal 1 by $\mathbf{1}_{n}$.

- Let $\delta(A)$ and $\omega(A)$ represent the matrices $\operatorname{diag}(v)$ and $A-\operatorname{diag}(v)$, respectively, where $v$ is formed by the elements of the main diagonal of $A$.

- For $a \in \mathbb{R}$ its sign is denoted by $\operatorname{sign}(a)$ and $|a|$ denotes its absolute value. For $v \in \mathbb{R}^{n}$, let $\operatorname{sign}(v)$ denote the vector $\left[\operatorname{sign}\left(v_{1}\right) \quad \ldots \quad \operatorname{sign}\left(v_{n}\right)\right]^{\top}$ and $|v|$ refer to the vector $\left[\begin{array}{lll}\left|v_{1}\right| & \ldots & \mid v_{n}\end{array}\right]^{\top}$.

- For a Lebesgue measurable function $u: \mathbb{R} \rightarrow \mathbb{R}^{m}$, define the norm $\|u\|_{\left(t_{1}, t_{2}\right)}=\operatorname{ess~sup}_{t \in\left(t_{1}, t_{2}\right)}\|u(t)\|$ for $\left(t_{1}, t_{2}\right) \subseteq \mathbb{R}$. We denote by $\mathscr{L}_{\infty}^{m}$ the space of functions $u$ with $\|u\|_{\infty}:=$ $\|u\|_{(-\infty,+\infty)}<+\infty$.

- A continuous function $\sigma: \mathbb{R}_{+} \rightarrow \mathbb{R}_{+}$belongs to class $\mathscr{K}$ if it is strictly increasing and $\sigma(0)=0$; it belongs to class $\mathscr{K}_{\infty}$ if it is also unbounded. A continuous function $\beta: \mathbb{R}_{+} \times \mathbb{R}_{+} \rightarrow \mathbb{R}_{+}$belongs to class $\mathscr{K} \mathscr{L}$ if $\beta(\cdot, r) \in \mathscr{K}$ and $\beta(r, \cdot)$ is a decreasing function going to zero for any fixed $r>0$.

- For a continuously differentiable function $V: \mathbb{R}^{n} \rightarrow \mathbb{R}$, denote by $\nabla V(v) f(v)$ the Lie derivative of $V$ along the vector field $f$ evaluated at point $v \in \mathbb{R}^{n}$.

\section{Preliminaries}

Definition 1: The matrix $A$ is said to be Hurwitz if all its eigenvalues lie in the open left half of the complex plane.

Definition 2: The matrix A is Metzler if all its off-diagonal entries are nonnegative.

Proposition 1 ([28]): Let $A \in \mathbb{R}^{n \times n}$ be Metzler. Then $A$ is Hurwitz if and only if there exists a vector $v>0$ in $\mathbb{R}^{n}$ with $A^{\top} v<0$.

Definition 3 ([15]): A continuous function $\Theta: \mathbb{R} \rightarrow \mathbb{R}^{n}$ is called almost periodic if, for every $\varepsilon>0$ there exists $L_{\varepsilon}>0$ such that every interval $\left[\theta, \theta+L_{\varepsilon}\right]$ for any $\theta \in \mathbb{R}$ contains at least one number $\eta$ for which

$$
\|\Theta(t+\eta)-\Theta(t)\|<\varepsilon
$$

for all $t \in \mathbb{R}$.

\section{A. Stability properties}

Consider the differential equation

$$
\begin{aligned}
& \dot{x}(t)=F(x(t), u(t)), t \geq t_{0} \in \mathbb{R}, \\
& y(t)=H(x(t)),
\end{aligned}
$$

where $x(t) \in \mathbb{R}^{n}$ is the state vector; $u(t) \in \mathbb{R}^{m}$ is the external input, $u \in \mathscr{L}_{\infty}^{m} ; y(t) \in \mathbb{R}^{p}$ is the output vector. Moreover, $F: \mathbb{R}^{n} \times \mathbb{R}^{m} \rightarrow \mathbb{R}^{n}$ is a continuous function and $H: \mathbb{R}^{n} \rightarrow \mathbb{R}^{p}$ is a continuously differentiable function. For an initial state $x_{0} \in \mathbb{R}^{n}$ and $u \in \mathscr{L}_{\infty}^{m}$, we denote the corresponding solution of the system (1) by $x\left(t, x_{0}, u\right)$ for the values of $t \in \mathbb{R}_{+}$the solution exists, so the corresponding output is $y\left(t, x_{0}, u\right)=$ $H\left(x\left(t, x_{0}, u\right)\right)$.

Definition 4: The system (1) is called forward complete if for all $x_{0} \in \mathbb{R}^{n}$ and $u \in \mathscr{L}_{\infty}^{m}$, the solution $x\left(t, x_{0}, u\right)$ is uniquely defined for all $t \geq t_{0}=0$.
Definition 5: A forward complete system (1) is said to be:

1) practically input-to-output stable (pIOS) if there exist $\beta \in \mathscr{K} \mathscr{L}, \gamma \in \mathscr{K}$ and $c \in \mathbb{R}_{+}$such that

$$
\left\|y\left(t, x_{0}, u\right)\right\| \leq \beta\left(\left\|x_{0}\right\|, t\right)+\gamma\left(\|u\|_{\infty}\right)+c, \forall t \in \mathbb{R}_{+}
$$

for any $x_{0} \in \mathbb{R}^{n}$ and $u \in \mathscr{L}_{\infty}^{m}$. The system is called input-to-output stable (IOS) if $c=0$. In the special case when $y=x$, the IOS property is called input-tostate stability (ISS).

2) robustly output stable (ROS) if there exist a smooth function $\alpha \in \mathscr{K}_{\infty}$ and $\beta \in \mathscr{K} \mathscr{L}$ such that the system

$$
\dot{x}=F(x, \kappa \alpha(\|H(x)\|))
$$

is forward complete, and the estimate

$$
\left\|y_{\alpha}\left(t, x_{0}, \kappa\right)\right\| \leq \beta\left(\left\|x_{0}\right\|, t\right), \forall t \in \mathbb{R}_{+}
$$

is satisfied for all $\kappa \in \mathscr{L}_{\infty}^{m}$ with $\|\kappa\|_{\infty} \leq 1$ and $y_{\alpha}\left(t, x_{0}, \kappa\right)=H\left(x\left(t, x_{0}, \kappa\right)\right)$ denotes the output function of the system (2).

Definition 6: A forward complete system (1) is said to be uniformly bounded-input-bounded-state stable (UBIBS) if there exists $\beta \in \mathscr{K}$ such that

$$
\left\|x\left(t, x_{0}, u\right)\right\| \leq \max \left\{\beta\left(\left\|x_{0}\right\|\right), \beta\left(\|u\|_{\infty}\right)\right\}, \forall t \in \mathbb{R}_{+}
$$

for all $x_{0} \in \mathbb{R}^{n}$ and $u \in \mathscr{L}_{\infty}^{m}$.

\section{B. Lyapunov characterizations}

Definition 7: For the system (1), a smooth function $V: \mathbb{R}^{n} \rightarrow \mathbb{R}_{+}$is

1) an ISS-Lyapunov function if there exist $\alpha_{1}, \alpha_{2}, \alpha_{3} \in$ $\mathscr{K}_{\infty}$ and $\chi \in \mathscr{K}$ such that

$$
\begin{gathered}
\alpha_{1}(\|x\|) \leq V(x) \leq \alpha_{2}(\|x\|) \\
\|x\| \geq \chi(\|u\|) \Rightarrow \nabla V(x) F(x, u) \leq-\alpha_{3}(\|x\|)
\end{gathered}
$$

for all $x \in \mathbb{R}^{n}$ and $u \in \mathbb{R}^{m}$.

2) an IOS-Lyapunov function if there exist $\alpha_{1}, \alpha_{2} \in \mathscr{K}_{\infty}$, $\chi \in \mathscr{K}$ and $\alpha_{3} \in \mathscr{K} \mathscr{L}$ such that

$$
\begin{gathered}
\alpha_{1}(\|H(x)\|) \leq V(x) \leq \alpha_{2}(\|x\|) \\
V(x) \geq \chi(\|u\|) \Rightarrow \nabla V(x) F(x, u) \leq-\alpha_{3}(V(x),\|x\|)
\end{gathered}
$$

for all $x \in \mathbb{R}^{n}$ and $u \in \mathbb{R}^{m}$.

3) a ROS-Lyapunov function if there exist $\alpha_{1}, \alpha_{2} \in \mathscr{K}_{\infty}$, $\chi \in \mathscr{K}$ and $\alpha_{3} \in \mathscr{K} \mathscr{L}$ such that

$$
\begin{gathered}
\alpha_{1}(\|H(x)\|) \leq V(x) \leq \alpha_{2}(\|x\|), \\
\|H(x)\| \geq \chi(\|u\|) \Rightarrow \nabla V(x) F(x, u) \leq-\alpha_{3}(V(x),\|x\|)
\end{gathered}
$$

for all $x \in \mathbb{R}^{n}$ and $u \in \mathbb{R}^{m}$.

Theorem 1 ([29]): The system (1) is ISS if and only if it admits an ISS-Lyapunov function.

Theorem 2 ([30]): A UBIBS system (1) is IOS (ROS) if and only if it admits an IOS (ROS)-Lyapunov function.

Remark 1: The UBIBS requirement can be dropped in the sufficient conditions of IOS or ROS if the system (1) is forward complete (or it possesses the unboundedness 
observability property [31]) and admits an IOS-Lyapunov function satisfying the conditions (4),

$$
V(x) \geq \chi(\|u\|) \Rightarrow \nabla V(x) F(x, u) \leq-\alpha_{3}(V(x))
$$

or a ROS-Lyapunov function satisfying the conditions (5),

$$
\|H(x)\| \geq \chi(\|u\|) \Rightarrow \nabla V(x) F(x, u) \leq-\alpha_{3}(V(x))
$$

for all $x \in \mathbb{R}^{n}$ and $u \in \mathbb{R}^{m}$, some $\alpha_{1}, \alpha_{2} \in \mathscr{K}_{\infty}$ and $\chi, \alpha_{3} \in \mathscr{K}$, respectively.

In the rest of the paper, to lighten the notation, the timedependency of variables might remain implicitly understood, for instance we will write $x$ for $x(t)$.

\section{Problem Statement}

Consider the following system in a generalized Persidskii form [25]:

$$
\dot{x}(t)=A_{0} x(t)+\sum_{j=1}^{M} A_{j} F_{j}(x(t))+\varphi(t), t \in \mathbb{R},
$$

where $x=\left[\begin{array}{lll}x_{1} & \ldots & x_{n}\end{array}\right]^{\top} \in \mathbb{R}^{n}$ is the state; $t_{0} \in \mathbb{R}$ denotes the initial time and $x\left(t_{0}\right)=x_{0}$; the disturbance $\varphi=$ $\left[\begin{array}{lll}\varphi_{1} & \ldots & \varphi_{n}\end{array}\right]^{\top} \in \mathscr{L}_{\infty}^{n}$

$$
F_{j}(x)=\left[\begin{array}{lll}
f_{j, 1}\left(x_{1}\right) & \ldots & f_{j, n}\left(x_{n}\right)
\end{array}\right]^{\top} \in \mathbb{R}^{n}, \forall j \in \overline{1, M} .
$$

Thus, the nonlinearity $F_{j}$ has a special structure: each element of $F_{j}$, the function $f_{j, r}$, depends on the coordinate $x_{r}$ only, $r \in \overline{1, n}$. We also require certain monotonicity of $F_{j}$ :

Assumption 1: Let the functions $f_{j, r}: \mathbb{R} \rightarrow \mathbb{R}, j \in \overline{1, M}, r \in$ $\overline{1, n}$ have the properties:

(i) $f_{j, r}$ are continuous and strictly increasing, $f_{j, r}(0)=0$;

(ii) $\lim _{a \rightarrow \pm \infty} f_{j, r}(a)= \pm \infty$.

Assumption 2: Assume that the matrices $A_{s} \in \mathbb{R}^{n \times n}, s \in$ $\overline{0, M}$ are Metzler and there exists a vector $v>0$ in $\mathbb{R}^{n}$ with $v^{\top} A_{s}=-c_{s}^{\top} \leq 0$ for $s \in \overline{0, M}$ and $\sum_{s=0}^{M} c_{s}>0$.

Note that we do not require that all matrices $A_{s}$ with $s \in$ $\overline{0, M}$ be Hurwitz, and only its total composition, evaluated by $\sum_{s=0}^{M} c_{s}$, has to provide the stability.

Definition 8 ([1]): System (8) is convergent if it admits a unique bounded solution for $t \in \mathbb{R}$ that is globally asymptotically stable.

Definition 9 ([18]): System (8) possesses almost periodic convergence property if it is convergent and the admitted solution is almost periodic.

The goal of this work is to formulate the conditions for (8) to be convergent, and if $\varphi$ is an almost periodic signal, to substantiate the same feature of the solutions.

\section{Conditions of CONVERGEnCE}

Note that for analysis of the convergence property in (8), we need to consider the system with $t \in \mathbb{R}$. However, the time dependence of the right-hand side of (8) comes through the input $\varphi$ only. As in the IOS/ISS framework, we can investigate the global stability of that system for $t \in \mathbb{R}_{+}$with $\varphi \in \mathscr{L}_{\infty}^{n}$, allowing us next to extend the obtained results to $t \in \mathbb{R}$. The main result of this paper is given below:

Theorem 3: If Assumptions 1 and 2 are fulfilled, then the system (8) is convergent.
Proof: The proof has two steps: first, the boundedness of solutions will be established using ISS theory; second, asymptotic convergence of different solutions of (8) will be shown.

1) Consider a Lyapunov function for the system (8):

$$
V(x)=v^{\top}|x|,
$$

where $v \in \mathbb{R}^{n}$ is given in Assumption 2. Since

$$
\min _{i \in \overline{1, n}} v_{i}\|x\| \leq V(x) \leq\|v\|\|x\|
$$

by Cauchy-Schwarz inequality, so the ISS condition (3) is verified.

Due to assumptions 1 and 2, it holds that the time derivative of $V$ is:

$$
\begin{aligned}
\dot{V}= & \operatorname{sign}^{\top}(x) \cdot \operatorname{diag}(v) \cdot\left(A_{0} x+\sum_{j=1}^{M} A_{j} F_{j}(x)+\varphi\right) \\
= & v^{\top} \operatorname{diag}\left(\operatorname{sign}^{\top}(x)\right)\left(\left(\delta\left(A_{0}\right)+\omega\left(A_{0}\right)\right) x\right. \\
& \left.+\sum_{j=1}^{M}\left(\delta\left(A_{j}\right)+\omega\left(A_{j}\right)\right) F_{j}(x)+\varphi\right) \\
\leq & v^{\top}\left(\delta\left(A_{0}\right)|x|+\omega\left(A_{0}\right)|x|+\sum_{j=1}^{M} \delta\left(A_{j}\right)\left|F_{j}(x)\right|\right. \\
& \left.+\sum_{j=1}^{M} \omega\left(A_{j}\right)\left|F_{j}(x)\right|+|\varphi|\right) \\
= & v^{\top}\left(A_{0}|x|+\sum_{j=1}^{M} A_{j}\left|F_{j}(x)\right|+|\varphi|\right) \\
\leq & -c_{0}^{\top}|x|-\sum_{j=1}^{M} c_{j}^{\top}\left|F_{j}(x)\right|+\|v\|\|\varphi\|_{\infty} .
\end{aligned}
$$

Thus, by Theorem 1 the system (8) is ISS (due to the imposed restrictions all nonlinearities are unbounded and the sum of vectors $c_{j}, j=\overline{0, M}$ is positive).

2) Next, along with (8), consider the system

$$
\dot{y}(t)=A_{0} y(t)+\sum_{j=1}^{M} A_{j} F_{j}(y(t))+\varphi(t)
$$

with the same input, but with different initial conditions $y\left(t_{0}\right) \in \mathbb{R}^{n}$. Define $z(t):=y(t)-x(t)$ for all $t \in \mathbb{R}_{+}$, then

$$
\dot{z}=A_{0} z+\sum_{j=1}^{M} A_{j} Q_{j}(x, z)
$$

where

$$
\begin{aligned}
Q_{j}(x, z) & =F_{j}(z+x)-F_{j}(x) \\
& =\left[\begin{array}{lll}
q_{j, 1}\left(x_{1}, z_{1}\right) & \ldots & q_{j, n}\left(x_{n}, z_{n}\right)
\end{array}\right]^{\top} \in \mathbb{R}^{n},
\end{aligned}
$$

$q_{j, r}\left(x_{r}, z_{r}\right)=f_{j, r}\left(z_{r}+x_{r}\right)-f_{j, r}\left(x_{r}\right)$ for all $j \in \overline{1, M}, r \in \overline{1, n}$. Note that for any $x \in \mathbb{R}^{n}$ the functions $Q_{j}$ satisfy the properties formulated in Assumption 1.

Consider a Lyapunov function

$$
\tilde{V}(z)=v^{\top}|z|,
$$


repeating the analysis made for (9), we see that $\tilde{V}$ is radially unbounded, $\tilde{V}>0$ for all $z \neq 0$ and $\tilde{V}(0)=0$. Again using Assumptions 1 and 2, we obtain

$$
\dot{\tilde{V}} \leq-c_{0}^{\top}|z|-\sum_{j=1}^{M} c_{j}^{\top}\left|F_{j}(z+x)-F_{j}(x)\right| .
$$

For any given number $l>0$, it holds that

$$
\dot{\tilde{V}} \leq-W_{l}(z)
$$

for $\|x\| \leq l, z \in \mathbb{R}^{n}$, where

$$
W_{l}(z)=\min _{\|x\| \leq l}\left[c_{0}^{\top}|z|+\sum_{j=1}^{M} c_{j}^{\top}\left|F_{j}(z+x)-F_{j}(x)\right|\right] .
$$

The proven properties of $\tilde{V}$ and $\dot{\tilde{V}}$ imply that all solutions of (10) satisfy $\|z(t)\| \leq \frac{\|v\|}{\min _{i \in \overline{1, n}} v_{i}}\left\|z\left(t_{0}\right)\right\|$ for all $z\left(t_{0}\right) \in \mathbb{R}^{n}$ and $t \in \mathbb{R}_{+}$. Applying LaSalle's invariance principle [14] we have

$$
\lim _{t \rightarrow+\infty}\|z(t)\|=0
$$

Therefore the system (8) is convergent.

Remark 2: It is also possible to use IOS/ROS properties to show the convergence for the system (10). Let

$$
H(z)=z
$$

be the output of (10) and consider IOS or ROS properties of the system (8), (10), (13). We see that the system (8), (10), (13) is with zero input, the conditions (5), (6) and (7) can be verified. Thus, all solutions of (10) are bounded by $\|z(t)\| \leq \beta\left(\left\|z\left(t_{0}\right)\right\|, t\right)$ for some $\beta \in \mathscr{K} \mathscr{L}$, all $z\left(t_{0}\right) \in \mathbb{R}^{n}$ and $t \in \mathbb{R}_{+}$.

The case when in the system (8) the input $\varphi$ is almost periodic is considered below:

Corollary 1: Let $\varphi \in \mathscr{L}_{\infty}^{n}$ be an almost periodic function for $t \in \mathbb{R}$, the functions $F_{j}, j \in \overline{1, M}$ be locally Lipschitz continuous and assumptions 1 and 2 be fulfilled, then the system (8) possesses almost periodic convergence property.

Proof: By Theorem 3, under assumptions 1 and 2 the system (8) is convergent with an almost periodic input $\varphi$. Therefore, the almost periodic convergence criterion proposed by Zubov [18] is fulfilled for the system (8).

\section{ApPlicAtion to MOdified LOTKA-VOLTERRA MODELS}

In this section, a modified Lotka-Volterra (LV) dynamics is considered, which has been widely investigated in infectious disease, biology, finance, to mention a few subjects [32]. However, the earliest basic model does not reflect some important phenomena, e.g., stable coexistence and predator preference. Therefore, there are many modified LV models. Among them, the following one additionally considers the mutualistic interactions in different species [33]:

$$
\dot{x}(t)=\operatorname{diag}(x(t))\left(r_{0}+r(t)+A x(t)+A_{2} \phi(x(t))\right), t \in \mathbb{R},
$$

where $x(t) \in \mathbb{R}_{+}^{n}$ is the population sizes of $n$ species; $r_{0} \in$ $\mathbb{R}^{n}$ denotes the birth or mortality rates of the species and $r(t) \in \mathbb{R}^{n}$ is introduced to model the deviations of the rates from the nominal quantities $r_{0} ; A \in \mathbb{R}^{n \times n}$ represents the community matrix and $A_{2} \in \mathbb{R}^{n \times n}$ refers to the net effect of the mutualism; the function $\phi: \mathbb{R}_{+}^{n} \rightarrow \mathbb{R}_{+}^{n}$, where $\phi_{i}(x)=$ $\frac{x_{i}}{b_{i}+x_{i}}$ with constants $b_{i}>0$ for $i \in \overline{1, n}$.

Assume there exists a unique equilibrium point $x_{e} \in \mathbb{R}_{+}^{n} \backslash$ $\{0\}$ for the dynamics (14) with $r(t)=0$ and define

$$
\rho(t)=\ln (x(t))-\ln \left(x_{e}\right)
$$

we obtain

$$
\dot{\rho}(t)=A_{1} F_{1}(\rho(t))+A_{2} F_{2}(\rho(t))+r(t),
$$

where

$$
\begin{gathered}
A_{1}:=A \operatorname{diag}\left(x_{e}\right), F_{1}(\rho)=e^{\rho}-\mathbf{1}_{n}, \\
F_{2}(\rho)=\phi\left(\operatorname{diag}\left(x_{e}\right) e^{\rho}\right)-\phi\left(x_{e}\right) .
\end{gathered}
$$

Note that for such functions $F_{1}$ and $F_{2}$, the requirement of Assumption 1 for $a \rightarrow-\infty$ is not satisfied. However, as in [23], due to the assumed existence of the global equilibrium $x_{e}$, it is possible to show that for sufficiently small $r$, all trajectories converge to a neighborhood of the steady-state, where the analysis can be next performed without taking into account the unbounded deviations of the state.

In the following examples, the convergence property for the system (15) with given values of $A, A_{2}, r_{0}$, and $r(t)$ is demonstrated with validation of the proposed conditions.

Example 1: Let

$$
\begin{gathered}
A=\left[\begin{array}{cc}
-0.8 & 0.4 \\
0.2 & -0.8
\end{array}\right], A_{2}=\left[\begin{array}{cc}
-0.5 & 0.3 \\
0.1 & -0.9
\end{array}\right], \\
b=\left[\begin{array}{l}
0.1 \\
0.4
\end{array}\right], r_{0}=\left[\begin{array}{l}
1 \\
0
\end{array}\right], r(t)=\left[\begin{array}{l}
\sin (t) \\
\cos (t)
\end{array}\right],
\end{gathered}
$$

where $r$ is a periodic signal representing the external input for (15), then the value of $x_{e}$ can be obtained as previously mentioned, and assumptions 1 and 2 are fulfilled. By choosing a periodic and oscillating input signal, we can see a convergence phenomenon not caused by the asymptotic stability of the considered system.

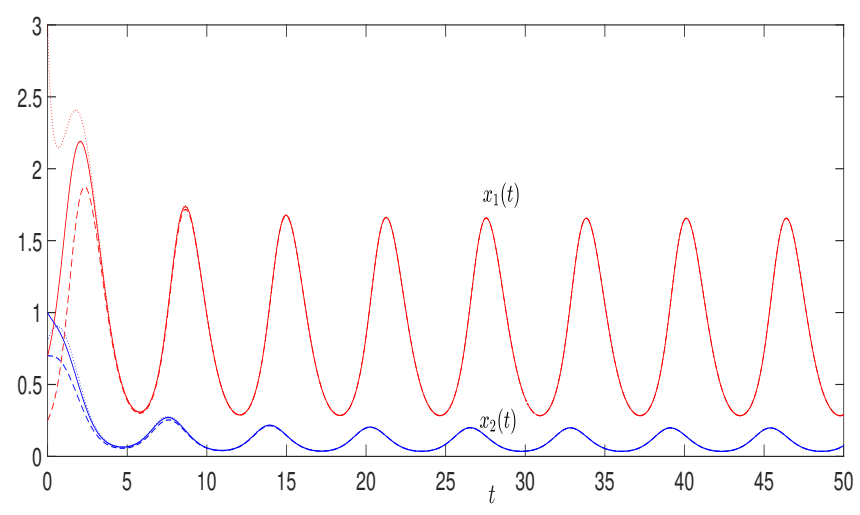

Fig. 1. The state trajectories versus the time $t$

Define the convergence error

$$
e=\left[\begin{array}{ccc}
I_{2} & -I_{2} & 0 \\
0 & I_{2} & -I_{2} \\
-I_{2} & 0 & I_{2}
\end{array}\right]\left[\begin{array}{l}
x^{1} \\
x^{2} \\
x^{3}
\end{array}\right]
$$




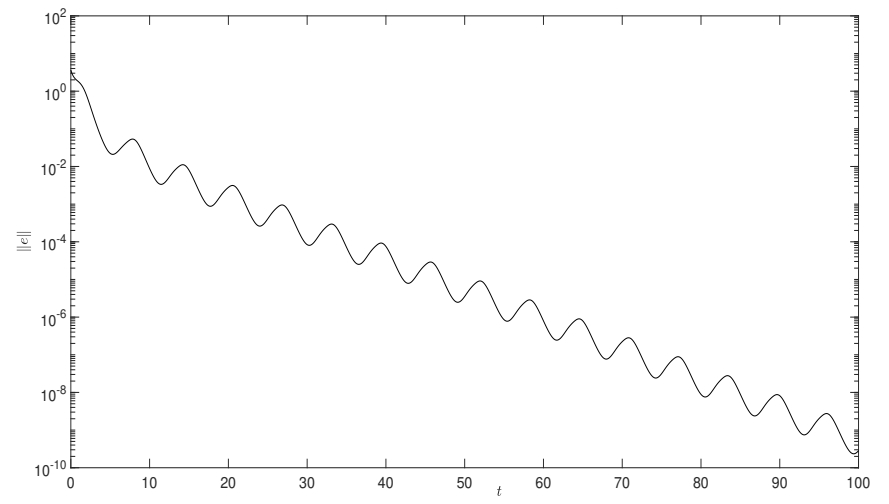

Fig. 2. The norms of the convergence errors $e$ versus the time $t$

where $x^{1}, x^{2}, x^{3} \in \mathbb{R}^{2}$ are the state trajectories issued from different initial conditions. Consider the trajectories with three distinct initial conditions:

$$
x^{1}(0)=\left[\begin{array}{c}
0.25 \\
0.7
\end{array}\right], x^{2}(0)=\left[\begin{array}{c}
3 \\
0.8
\end{array}\right], x^{3}(0)=\left[\begin{array}{c}
0.7 \\
1
\end{array}\right] .
$$

The state transients and the norms of the convergence errors $e$ are shown in Fig. 1 and Fig. 2, respectively. Note that $x_{1}$ and $x_{2}$ in Fig. 1 refer to the first and second state variables of $x^{1}, x^{2}, x^{3}$, respectively. The trajectory in Fig. 2 represents the values of $\|e\|$ corresponding to the state values in Fig. 1. These results demonstrate the convergence phenomenon in (15).

Example 2: Consider the case with the same $A, A_{2}, b$, $x^{1}(0), x^{2}(0), x^{3}(0)$ as previously, while the value of $r_{0}$ and the rate deviation $r$ are revised to

$$
r_{0}=\left[\begin{array}{c}
0.8 \\
0
\end{array}\right], r(t)=\left[\begin{array}{c}
\sin (t)+\sin (\sqrt{2} t) \\
\cos (t)+\cos (\sqrt{2} t)
\end{array}\right],
$$

then such an external input is almost periodic, which allows us to study the almost-periodic convergence property of the system in this example (see Corollary 1).

Fig. 3 and Fig. 4 present the corresponding state trajectories and the values of $\|e\|$ versus the time $t$, respectively. These simulation results also demonstrate the convergence property in (15), which is almost periodic in this case.

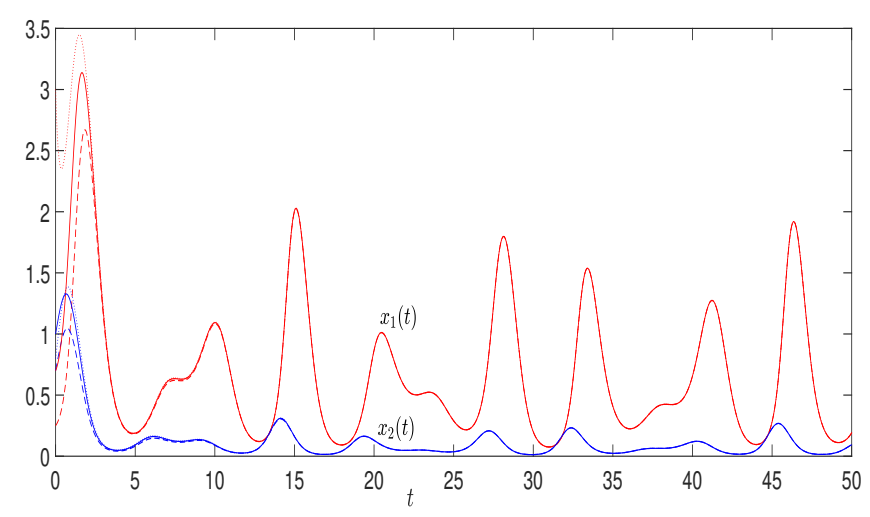

Fig. 3. The state trajectories versus the time $t$ (the almost-periodic case)

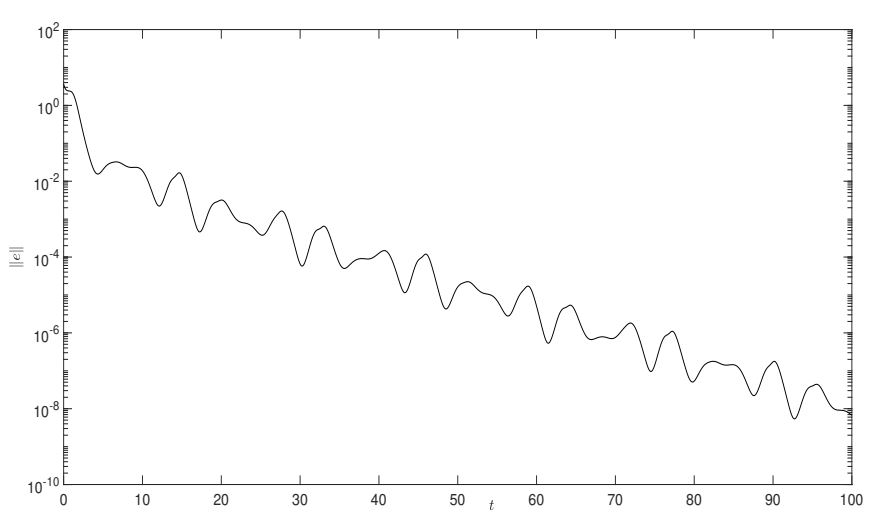

Fig. 4. $\|e\|$ versus the time $t$ (the almost-periodic case)

\section{CONCLUSION}

In this paper, the convergence and almost periodic convergence conditions for generalized Persidskii systems were proposed. The formulated conditions were obtained in the form of linear algebraic inequalities. Therefore, they can be constructively verified. Simulations with Lotka-Volterra models were presented for the examination of the proposed results.

The future research directions include the analysis of a more sophisticated Lyapunov function than (9) and investigation of the robust convergence properties.

\section{REFERENCES}

[1] A. Pavlov, N. Wouw, and H. Nijmeijer, "Convergent systems: Analysis and synthesis," in Control and Observer Design for Nonlinear Finite and Infinite Dimensional Systems. Berlin/Heidelberg: SpringerVerlag, pp. 131-146.

[2] A. Pavlov and N. van de Wouw, "Convergent systems: Nonlinear simplicity," ser. 2017 Lecture Notes in Control and Information Sciences, 2017, vol. 470, pp. 51-77.

[3] D. Angeli, "A lyapunov approach to incremental stability properties," IEEE Transactions on Automatic Control, vol. 47, pp. 410-421, 2002.

[4] B. S. Rüffer, N. van de Wouw, and M. Mueller, "Convergent systems vs. incremental stability," Systems \& Control Letters, vol. 62, pp. 277 $285,2013$.

[5] J. Jouffroy and T. Fossen, "Tutorial on incremental stability analysis using contraction theory," Modeling, Identification and Control: A Norwegian Research Bulletin, vol. 31, pp. 93-106, 2010.

[6] A. Pavlov, N. van de Wouw, and H. Nijmeijer, Uniform Output Regulation of Nonlinear Systems. Boston, MA: Birkhäuser Boston, 2006.

[7] A. Y. Pogromsky, "Passivity based design of synchronizing systems," International Journal of Bifurcation and Chaos, vol. 08, pp. 295-319, 1998

[8] C. Cai and G. Chen, "Synchronization of complex dynamical networks by the incremental ISS approach," Physica A: Statistical Mechanics and its Applications, vol. 371, pp. 754-766, 2006.

[9] G.-B. Stan and R. Sepulchre, "Analysis of interconnected oscillators by dissipativity theory," IEEE Transactions on Automatic Control, vol. 52, pp. 256-270, 2007.

[10] A. Pavlov, N. van de Wouw, and H. Nijmeijer, "Frequency response functions for nonlinear convergent systems," IEEE Transactions on Automatic Control, vol. 52, pp. 1159-1165, 2007.

[11] R.-W. Liu, "Convergent systems," IEEE Transactions on Automatic Control, vol. 13, pp. 384-391, 1968.

[12] A. A. Kosov, "Investigation of convergence of large scale almost periodic systems by means of comparison vector functions with components as forms of even degrees," Russian Mathematics, vol. 59, no. 7, pp. 21-30, 2015. 
[13] A. A. Kosov and V. N. Shchennikov, "On the convergence phenomenon in complex almost periodic systems," Differential Equations, vol. 50, no. 12, pp. 1573-1583, 2014.

[14] H. Khalil, Nonlinear systems. Upper Saddle River, NJ: Prentice-Hall, 2002.

[15] B. Levitan and V. Zhikov, Almost periodic funcitons and differential equations. New York: Cambridge University Press, 1982.

[16] V. A. Pliss, Nonlocal problems of the theory of oscillations. London: Academic Press, 1966.

[17] A. Pavlov, A. Pogromsky, N. van de Wouw, and H. Nijmeijer, "Convergent dynamics, a tribute to Boris Pavlovich Demidovich," Systems \& Control Letters, vol. 52, pp. 257-261, 2004.

[18] V. I. Zubov, Theory of Oscillations. World Scientific, 1999.

[19] - Oscillations in Nonlinear and Controlled Systems. Leningrad: Sudpromgiz, 1962 (in Russian).

[20] T. Yoshizawa, Stability theory by Lyapunov's second method. Mathematical Society of Japan, 1966.

[21] V. A. Yakubovich, "Method of matrix unequalities in theory of nonlinear control systems stability. I. forced oscillations absolute stability," Avtomat. i Telemekh., vol. 25, pp. 1017-1029, 1964.

[22] T. G. Hallam, "Convergence of solutions of perturbed nonlinear differential equations," Annali di Matematica Pura ed Applicata, vol. 94, pp. 275-282, 1972.

[23] A. Aleksandrov and E. Aleksandrova, "Convergence conditions for some classes of nonlinear systems," Systems \& Control Letters, vol. 104, pp. 72-77, 2017.

[24] E. Barbashin, "On construction of Lyapunov functions for nonlinear systems," in Proc. 1st IFAC World Congress, Moscow, 1961, pp. 742751.

[25] S. Persidskii, "Concerning problem of absolute stability," Automation and Remote Control, pp. 5-11, 1969.

[26] D. Efimov and A. Aleksandrov, "Robust stability analysis and implementation of Persidskii systems," 58th IEEE Conference on Decision and Control $(C D C), 2019$.

[27] W. Mei, D. Efimov, and R. Ushirobira, "Feedback synchronization in Persidskii systems," 21th IFAC World Congress, 2020.

[28] E. Kaszkurewicz and A. Bhaya, Matrix Diagonal Stability in Systems and Computation. Boston, MA: Birkhäuser Boston, 2000.

[29] D. Sontag and Y. Wang, "On characterizations of input-to-state stability with respect to compact sets," IFAC Proceedings Volumes, vol. 28, pp. 203-208, 1995.

[30] E. Sontag and Y. Wang, "Lyapunov characterizations of input to output stability," SIAM Journal on Control and Optimization, vol. 39, no. 1, pp. 226-249, 2000.

[31] D. Angeli and E. Sontag, "Forward completeness, unboundedness observability, and their Lyapunov characterizations," Systems \& Control Letters, vol. 38, pp. 209-217, 1999.

[32] R. D. Holt and J. Pickering, "Infectious disease and species coexistence: A model of Lotka-Volterra form," The American Naturalist, vol. 126, pp. 196-211, 1985.

[33] J. N. Holland, D. L. DeAngelis, and J. L. Bronstein, "Population dynamics and mutualism: Functional responses of benefits and costs," The American Naturalist, vol. 159, pp. 231-244, 2002. 